\title{
The relationship between neutrophil to lymphocyte ratio, platelet to lymphocyte ratio and thrombolysis in myocardial infarction risk score in patients with ST elevation acute myocardial infarction before primary coronary intervention
}

\author{
Halit Acet ${ }^{1}$, Faruk Ertaş ${ }^{1}$, Mehmet Zihni Bilik ${ }^{1}$, Mehmet Ata Akıl ${ }^{1}$, Ferhat Özyurtlu², Mesut Aydın ${ }^{1}$, \\ Mustafa Oylumlu ${ }^{1}$, Nihat Polat ${ }^{1}$, Murat Yüksel ${ }^{1}$, Abdulkadir Yıldız $^{1}$, Hasan Kaya ${ }^{1}$, Abdurrahman Akyüz $^{1}$, \\ Hilal Ayçiçek ${ }^{1}$, Mehmet Özbek ${ }^{1}$, Nizamettin Toprak ${ }^{1}$ \\ ${ }^{1}$ Department of Cardiology, Faculty of Medicine, Dicle University, Diyarbakır, Turkey \\ ${ }^{2}$ Department of Cardiology, Faculty of Medicine, İzmir University, İzmir, Turkey
}

Postep Kardiol Inter 2015; 11, 2 (40): 126-135

DOI: $10.5114 /$ pwki.2015.52286

\begin{abstract}
A bstract
Introduction: The thrombolysis in myocardial infarction (TIMI) risk score is calculated as the sum of independent predictors of mortality and ischemic events in ST elevation acute myocardial infarction (STEMI). Several studies show that the neutrophil to lymphocyte ratio (NLR) is a prognostic inflammatory marker. In preliminary studies, platelet to lymphocyte ratio (PLR) has been proposed as a pro-thrombotic marker. The relationship between NLR, PLR and TIMI risk score for STEMI has never been studied.

Aim: To evaluate the association between TIMI-STEMI risk score and NLR, PLR and other biochemical indices in STEMI.

Material and methods: In this retrospective study, we evaluated 390 patients who presented with STEMI within $12 \mathrm{~h}$ of symptom onset. Patients were grouped according to low and high TIMI risk scores.

Results: We enrolled 390 patients (mean age $61.9 \pm 13.6$ years; $73 \%$ were men). The NLR, platelet distribution width (PDW) and uric acid level (UA) were significantly associated with a high TIMI-STEMI risk score $(p=0.016, p=0.008, p=0.030$, respectively), but PLR was not associated with a high TIMI-STEMI risk score. Left ventricular ejection fraction was an independent predictor of TIMI-STEMI risk score. A cut-off point of TIMI-STEMI score of > 4 predicted in-hospital mortality (sensitivity $75 \%$, specificity $70 \%, p$ $<0.001$ ). We found that NLR, PDW, and UA level were associated with TIMI-STEMI risk score.

Conclusions: Neutrophil to lymphocyte ratio, PDW and UA level are convenient, inexpensive and reproducible biomarkers for STEMI prognosis before primary angioplasty when these indicators are combined with the TIMI-STEMI risk score. We believe that these significant findings can guide further clinical practice.
\end{abstract}

Key words: TIMI risk score, hematologic indices, STEMI, platelet to lymphocyte ratio, neutrophil to lymphocyte ratio.

\section{Introduction}

Coronary artery disease (CAD) and acute myocardial infarction (MI) are a major cause of death and morbidity worldwide. Atherosclerosis is the major cause of cardiovascular disease (CVD) [1, 2]. Coronary atherosclerosis is the main cause of ST elevation acute myocardial infarction (STEMI). Multiple pathophysiological factors influence this atherosclerotic process, and one of the most important factors is inflammation [3, 4]. The inflamma- tory process that underlines atherosclerosis has a critical role in plaque destabilization. This process also affects the development of thrombus that is superimposed on the erosion of an atherosclerotic plaque and consequently causes MI [5]. Reperfusion therapy, either pharmacological or mechanical, is indicated in patients with STEMI with duration of less than $12 \mathrm{~h}$. The superiority of primary percutaneous coronary intervention ( $\mathrm{PPCl}$ ) over fibrinolysis has been demonstrated in several studies $[6,7]$.

\section{Corresponding author:}

Halit Acet MD, Assist. Prof., Department of Cardiology, Faculty of Medicine, Dicle University, Diyarbakır, phone: +90 4122488001 , +90 5053190183, fax: +90 412 2488440, e-mail: halitacet@gmail.com

Received: 6.03.2015, accepted: 26.03.2015. 
However, it has been observed that the benefit of PPCI is different in each group of patients and the benefit is greatest in those at high risk [8]. Thus, risk stratification prior to intervention has great clinical importance to identify patients at higher risk and to optimize their therapeutic management. The risk scores applied to patients who are treated exclusively with PPCI have shown favorable results $[9,10]$. The thrombolysis in myocardial infarction (TIMI) risk score for STEMI was calculated as the sum of independent predictors of mortality and ischemic events [11] and derived from STEMI patients in the In TIME II randomized controlled trial [12].

Several studies have shown that the neutrophil to lymphocyte ratio (NLR) is a prognostic marker in patients with CAD $[13,14]$. Platelets are a source of inflammatory mediators [15]. However, platelets have a major effect on the formation of atherothrombosis, and therefore play an important role in the pathogenesis of acute coronary syndrome (ACS) [16]. The association between low lymphocyte count and major adverse cardiovascular outcomes (MACE) has also been shown in several studies $[17,18]$. Recently, the platelet to lymphocyte ratio (PLR) has been proposed to be a pro-thrombotic and inflammatory marker [18-20]. The relationship between NLR, PLR and TIMI risk score in patients with STEMI has never been studied.

\section{Aim}

In this study, we investigated the association between TIMI risk score and NLR, PLR and other biochemical indices in patients with STEMI undergoing PPCI.

\section{Material and methods}

\section{Study design and patients}

We evaluated 750 patients who retrospectively presented with STEMI and underwent PPCI within $12 \mathrm{~h}$ of symptom onset between January 2012 and January 2014. We defined STEMI based on criteria created by the American College of Cardiology and the European Society of Cardiology [21] as: an increase in troponin I > $1 \mathrm{ng} / \mathrm{ml}$; a new ST elevation as measured from the J-point in 2 or more contiguous leads from leads V1, V2, and V3 measuring at least $0.2 \mathrm{mV}$ or at least $0.1 \mathrm{mV}$ in the remaining leads during the first $12 \mathrm{~h}$ after symptom onset or newly developed left bundle branch block (LBBB) pattern.

Patients with severe liver disease, autoimmune diseases, cancer, hematological disorders, severe valvular disease, inflammatory, and infectious diseases were excluded from the study. Patients on the following medications were excluded from the study: thrombolytic therapy and glycoprotein Ilb/IIla inhibitors. If at any point during the study the patient was not treated with $\mathrm{PPCl}$, did not follow up for blood work, or had poor echocardiographic windows, they were also eliminated from the investigation. After accounting for all of these exclusion criteria, a total of 390 patients remained in the study sample. All patients received a complete physical examination and assessment of coronary risk factors. We recorded medical histories and presenting clinical symptoms. Patients were also evaluated according to Killip clinical examination classification [22].

Demographic data and variables determined TIMI risk score points. A total score of 0-14 was possible according to the following characteristics: age, diabetes mellitus (DM)/hypertension (HT) or angina, heart rate $<100$ bpm, systolic blood pressure < $100 \mathrm{mmHg}$, Killip class II-IV, weight $<67 \mathrm{~kg}$, anterior MI or LBBB presentation, latency $>4 \mathrm{~h}$ [11]. Calculation of the TIMI risk score was performed with a computer program (http://www.mdcalc. com/timi-risk-score-for-stemi/). Patients were classified as low risk if their TIMI score was $\leq 4$ points (group I) and as high risk if their TIMI score was $>4$ points (group II). The in-hospital death Global Registry of Acute Coronary Events (GRACE) risk score points (which include age, creatinine, heart rate, systolic blood pressure, Killip class, cardiac arrest at admission, elevated cardiac markers, and ST-segment deviation) were recorded [23], and calculation of the GRACE risk score was performed using a computer program (www.outcomesumassmed.org/ grace/acs_risk/acs_risk_content.html).

Monitoring for MACE was performed during the in-hospital follow-up period. Examples of MACE were acute stent thrombosis, cardiogenic shock, new advanced heart failure, pulmonary edema, complete atrioventricular block (AVB) requiring a temporary pacemaker, severe ventricular arrhythmia and in-hospital mortality during the post-PPCl follow-up period. An in-hospital death was only considered a MACE if the death was due to myocardial infarction, cardiac arrest, or some other cardiac-related cause.

Acute stent thrombosis was defined as an abrupt onset of cardiac symptoms after stent deployment within the first $24 \mathrm{~h}$ with evidence of a flow-limiting thrombus on angiography near the newly placed stent. Cardiogenic shock was defined as marked and persistent hypotension lasting more than 30 min with a systolic arterial pressure less than $80 \mathrm{~mm} \mathrm{Hg}$ with signs of hypoperfusion due to left ventricular dysfunction, right ventricular infarction, or cardiac mechanical complications. New-onset advanced heart failure was diagnosed if the patient qualified for a New York Heart Association functional classification of III or greater. In order for a severe ventricular arrhythmia to be considered a MACE, it needed to occur within $48 \mathrm{~h}$ of admission, and the rhythm had to be ventricular fibrillation, ventricular tachycardia, or asystole.

Transthoracic echocardiography was performed upon admission to determine left ventricular ejection fraction (LVEF) (Vivid S6, GE Medical Systems, USA). All patients underwent selective coronary angiography using the Judkins technique. We performed PPCI with the standard 
femoral approach using a $7 \mathrm{Fr}$ guiding catheter. For all study participants, only one artery was identified as the culprit lesion. Coronary vessel disease was defined as greater than a $50 \%$ stenosis in one of the major coronary arteries. The Gensini scoring system was used to determine the severity of CAD [24].

\section{Laboratory analysis}

On admission, we obtained venous blood from all patients. The NLR was calculated as the ratio of the neutrophils and lymphocytes, both obtained from the same automated blood sample at admission. Baseline PLR was measured by dividing the platelet count by the lymphocyte count. Hematologic indices were measured by an automated hematology analyzer system (Abbott Cell-Dyn 3700; Abbott Laboratory, Abbott Park, Illinois). Absolute cell counts were utilized to perform subsequent analyses. Total cholesterol, high-density lipoprotein (HDL), low-density lipoprotein (LDL), triglycerides, glucose, uric acid level (UA) and creatinine levels were measured with the Abbott Architect C16000 autoanalyzer (Abbott Laboratory). Fasting lipid panels were obtained after an overnight fast.

\section{Statistical analysis}

Statistical analyses were performed using the SPSS software version 18.0 (SPSS Inc., Chicago, Illinois, USA). Categorical variables were summarized as percentages and compared with the Pearson $\chi^{2}$ test. Continuous variables were presented as mean \pm standard deviation and tested for normal distribution by the Kolmogorov-Smirnov test. Comparative analyses between groups were performed using the $\chi^{2}$ or Fisher's exact test and independent samples $t$-test or Mann-Whitney $U$-test, when appropriate. Spearman's test was used for correlation analysis between TIMI risk score for STEMI and other parameters. Multivariate stepwise forward logistic regression analysis was used to assess independent predictors of TIMI risk score for STEMI. All variables that were found to be significant in univariate analysis were included in the logistic regression model, and results are shown as an odds ratio (OR) with $95 \%$ confidence intervals $(\mathrm{CI})$. A $p$-value less than 0.05 was considered significant. The cut-off points for sensitivity and specificity of TIMI risk score for STEMI in predicting in-hospital mortality were estimated by performing a receiver operator characteristic (ROC) curve analysis.

The study protocol was reviewed and approved by the Ethics Committee in accordance with the Declaration of Helsinki.

\section{Results}

We obtained data from a total of 756 patients with STEMI, who were taken to the catheterization laboratory to undergo $\mathrm{PPCl}$. After accounting for exclusion criteria,
390 patients were included in the data analysis. The distribution of patients according to TIMI score was as follows: 1 point, 49 patients (12.6\%); 2 points, 74 patients (19.0\%); 3 points, 51 patients (13.1\%); 4 points, 78 patients $(20.0 \%) ; 5$ points, 44 patients $(11.3 \%) ; 6$ points, 30 patients $(7.7 \%) ; 7$ points, 31 patients $(7.9 \%)$; and $\geq 8$ points, 33 patients $(8.4 \%)$. The study population was divided into two groups according to TIMI risk scores as: group I $(\leq 4, n=252)$ and group II (>4, $n=138)$. Mean TIMI risk scores for groups I and II were 2.6 and 6.8 , respectively. Comparisons of the baseline characteristics of the groups are shown in Table I.

The average age of the population was $61.93 \pm 13.62$ years and $72.6 \%$ were men. In comparison to group I, patients in group II were older. There were no significant differences regarding known atherogenic risk factors and previous medications, but smoking was greater in group I ( $p<0.001)$. Killip class II-IV designations were more common in group II $(p<0.001)$. The majority of group II patients had an anterior MI caused by an occlusion of the left anterior descending coronary artery (LAD). Moreover, patients in group II showed higher rates of multivessel disease $(p=0.018)$. Patients in group II had lower LVEF measurements than group I $(p<0.001)$. Duration of hospitalization was significantly longer in group II patients $(p=0.001)$. The Gensini and GRACE scores were higher in group II when compared to group I $(p=0.001, p<0.001)$. Initial laboratory findings are shown in Table II.

In comparison to group I, glucose on admission $(p=0.013)$, creatinine on admission $(p=0.001)$, UA ( $p=$ $=0.030), \operatorname{NLR}(p=0.016)$ and PDW $(p=0.008)$ values (Figure 1) for patients in group II were significantly greater than those of group I. The was no significant difference between groups for PLR and fasting lipid panels.

Adverse events that occurred in both groups during hospitalization are shown in Table III. We observed that in-hospital mortality $(p<0.001)$ and MACE $(p<0.001)$ were significantly higher in patients of group II. Similarly, other adverse events also occurred more frequently in group II. In-hospital advanced heart failure ( $p<0.001)$, advanced pulmonary edema $(p<0.001)$, development cardiogenic shock $(p<0.001)$ and serious ventricular arrhythmias $(p<0.001)$ were more common in group II patients.

Spearman's correlation analysis revealed significant associations between age $(r=0.626, p<0.001)$, heart rate $(r=0.271, p<0.001)$, latency $(r=0.313, p \leq 0.001)$, creatinine on admission $(r=0.244, p<0.001)$, glucose on admission $(r=0.169, p<0.001)$, LVEF $(r=-0.440$, $p<0.001)$, GRACE risk score $(r=0.736, p<0.001)$, Killip class $(r=0.271, p<0.001)$, NLR $(r=0.113, p=0.032)$, PDW $(r=0.110, p=0.029)$ and TIMI score.

Stepwise forward multivariable logistic regression analysis was used to assess the independent association of clinical parameters with high TIMI risk score. Older age 
Table I. Demographic characteristics of patients according to TIMI-STEMI risk score groups

\begin{tabular}{|c|c|c|c|}
\hline Variables & $\begin{array}{c}\text { TIMI } \leq 4 \text { points } \\
n=252\end{array}$ & $\begin{array}{c}\text { TIMI > } 4 \text { points } \\
n=138\end{array}$ & Value of $p$ \\
\hline Age [years] & $56.85 \pm 11.73$ & $71.20 \pm 11.83$ & $<0.001^{* *}$ \\
\hline Sex, male, $n(\%)$ & $201(80)$ & $82(59)$ & $<0.001$ \\
\hline \multicolumn{4}{|l|}{ Previous history: } \\
\hline Hypertension, $n(\%)$ & $86(34)$ & $55(40)$ & 0.260 \\
\hline Diabetes mellitus, $n(\%)$ & $63(25)$ & $36(26)$ & 0.814 \\
\hline Smoking, $n(\%)$ & $159(63)$ & $58(42)$ & $<0.001$ \\
\hline Hyperlipidemia, $n(\%)$ & $13(5)$ & $7(5)$ & 0.971 \\
\hline Family history of CAD, $n(\%)$ & $63(25)$ & $15(11)$ & 0.001 \\
\hline MI or CAD, $n(\%)$ & $12(5)$ & $8(6)$ & 0.658 \\
\hline $\mathrm{PCl}, n(\%)$ & $15(6)$ & $7(5)$ & 0.719 \\
\hline \multicolumn{4}{|l|}{ Previous medications: } \\
\hline Preadmission aspirin use, $n(\%)$ & $192(76)$ & $104(75)$ & 0.855 \\
\hline Preadmission clopidogrel use, $n(\%)$ & $126(50)$ & $65(47)$ & 0.584 \\
\hline$\beta$-Blocker, $n(\%)$ & $29(12)$ & $12(9)$ & 0.399 \\
\hline ACE inhibitors, $n(\%)$ & $28(11)$ & $13(9)$ & 0.603 \\
\hline Statin, $n(\%)$ & $16(6)$ & $13(9)$ & 0.269 \\
\hline Preadmission enoxaparin use, $n(\%)$ & $231(92)$ & $118(35)$ & 0.058 \\
\hline \multicolumn{4}{|l|}{ Killip class on presentation, $n(\%)$} \\
\hline 1 & $234(93)$ & $60(44)$ & $<0.001$ \\
\hline II & $16(6.2)$ & $47(34)$ & \\
\hline III & $1(0.4)$ & $14(10)$ & \\
\hline IV & $1(0.4)$ & $17(12)$ & \\
\hline Admission SBP [mm Hg] & $129.13 \pm 21.09$ & $123.82 \pm 29.30$ & $0.062^{*}$ \\
\hline Admission heart rate [bpm] & $80.52 \pm 14.99$ & $88.37 \pm 19.52$ & $<0.001^{\star}$ \\
\hline \multicolumn{4}{|l|}{ Location of STEMI: } \\
\hline Anterior, $n(\%)$ & $92(37)$ & $88(64)$ & $<0.001$ \\
\hline Non-anterior, $n(\%)$ & $160(63)$ & $50(36)$ & \\
\hline Latency $[\mathrm{h}]$ & $5.08 \pm 3.21$ & $7.18 \pm 3.50$ & $<0.001^{\star \star}$ \\
\hline Admission LVEF (\%) & $46.22 \pm 9.36$ & $37.96 \pm 10.62$ & $<0.001^{\star \star}$ \\
\hline \multicolumn{4}{|l|}{ Location of culprit lesion: } \\
\hline LAD, $n(\%)$ & $97(39)$ & $88(64)$ & $<0.001$ \\
\hline RCA, $n(\%)$ & $117(46)$ & $30(22)$ & \\
\hline $\mathrm{CX}, n(\%)$ & $38(15)$ & $19(14)$ & \\
\hline \multicolumn{4}{|l|}{ Number of narrowed coronary arteries: } \\
\hline 1 vessel, $n(\%)$ & $117(46)$ & $47(34)$ & 0.018 \\
\hline$>1$ vessel, $n(\%)$ & $135(54)$ & $91(66)$ & \\
\hline Gensini score & $56.07 \pm 28.32$ & $68.10 \pm 34.59$ & $0.001^{\star *}$ \\
\hline GRACE risk score & $138.75 \pm 27.59$ & $192.49 \pm 41.69$ & $<0.001^{\star \star}$ \\
\hline Duration of hospitalization [days] & $5.03 \pm 4.5$ & $6.54 \pm 7.29$ & $0.001^{\star *}$ \\
\hline
\end{tabular}

${ }^{*}$ Student's t-test, **Mann-Whitney U-test, For other statistics $\chi^{2}$ test, MI - myocardial infarction, CAD - coronary artery disease, PCI - percutaneous coronary intervention, $A C E$ - angiotensin converting enzyme, SBP - systolic blood pressure, $L V E F$ - left ventricular ejection fraction, $L A D$ - left anterior descending coronary artery, RCA - right coronary artery, CX - circumflex coronary artery, TIMI - thrombolysis in myocardial infarction, GRACE - Global Registry of Acute Coronary Events.

$(b=0.057$, odds ratio $(\mathrm{OR}) 1.059,95 \% \mathrm{Cl}: 1.023-1.095$, $p=0.001)$, increased heart rate $(b=0.028, \mathrm{OR}=1.028$, $95 \% \mathrm{Cl}: 1.006-1.051, p=0.012)$ latency $(b=0.214$, $\mathrm{OR}=1.239,95 \% \mathrm{Cl}: 1.118-1.373, p<0.001)$, LVEF on admission $(b=-0.060, \mathrm{OR}=0.942,95 \% \mathrm{Cl}: 0.911-0.974$, $p<0.001)$, creatinine on admission $(b=-0.745, \mathrm{OR}=0.475$, 95\% Cl: $0.247-0.912, p=0.025)$, and higher GRACE risk score $(b=0.049, \mathrm{OR}=1.050,95 \% \mathrm{Cl}: 1.033-1.068$, $p<0.001)$ were independently associated with a higher TIMI risk score for STEMI.

In ROC analysis, a cut-off point of $>4$ TIMI score predicted in-hospital mortality (sensitivity $75.0 \%$, specificity $69.7 \%$, and area under curve (AUC) $0.782,95 \% \mathrm{Cl}: 0.705-$ $0.859 ; p<0.001)$. 
Table II. Biochemical findings of patients according to TIMI-STEMI risk score groups

\begin{tabular}{|c|c|c|c|}
\hline Variables & TIMI $\leq 4$ points & TIMI > 4 points & Value of $p$ \\
\hline White blood cell count $[K / \mu l]$ & $13.25 \pm 4.60$ & $14.18 \pm 6.57$ & 0.147 \\
\hline Neutrophil count & $10.48 \pm 4.35$ & $11.39 \pm 6.05$ & 0.122 \\
\hline Lymphocyte count & $2.03 \pm 1.24$ & $1.89 \pm 1.18$ & 0.272 \\
\hline Neutrophil to lymphocyte ratio & $6.70 \pm 4.17$ & $8.36 \pm 7.07$ & 0.016 \\
\hline Platelet count $[\mathrm{K} / \mu \mathrm{ll}]$ & $251.43 \pm 62.36$ & $249.09 \pm 70.32$ & 0.461 \\
\hline Platelet to lymphocyte ratio & $157.63 \pm 84.71$ & $180.06 \pm 133.04$ & 0.087 \\
\hline Mean platelet volume & $8.16 \pm 1.49$ & $8.39 \pm 1.44$ & 0.144 \\
\hline Red cell distribution width & $15.89 \pm 1.53$ & $16.18 \pm 1.51$ & 0.08 \\
\hline Platelet distribution width & $17.76 \pm 1.08$ & $18.13 \pm 1.34$ & 0.008 \\
\hline Monocyte count & $0.65 \pm 0.30$ & $0.70 \pm 0.35$ & 0.205 \\
\hline Basophil count & $0.07 \pm 0.05$ & $0.07 \pm 0.06$ & 0.539 \\
\hline Red blood cell count $[\mathrm{M} / \mu \mathrm{l}]$ & $4.95 \pm 0.57$ & $4.73 \pm 0.58$ & $<0.001^{*}$ \\
\hline Hemoglobin $[\mathrm{g} / \mathrm{dl}]$ & $14.02 \pm 1.65$ & $13.49 \pm 1.65$ & $0.002^{*}$ \\
\hline Glucose on admission [mg/dl] & $170.26 \pm 89.06$ & $192.89 \pm 100.77$ & $0.013^{*}$ \\
\hline Creatinine on admission [mg/dl] & $0.93 \pm 0.77$ & $1.04 \pm 0.53$ & $0.001^{*}$ \\
\hline Uric acid $[\mathrm{mg} / \mathrm{dl}]$ & $6.72 \pm 2.57$ & $7.24 \pm 2.60$ & $0.030^{\star}$ \\
\hline \multicolumn{4}{|l|}{ Fasting lipid panel: } \\
\hline Total cholesterol [mg/dl] & $178.99 \pm 41.32$ & $184.30 \pm 48.99$ & 0.260 \\
\hline Low-density lipoprotein [mg/dl] & $114.30 \pm 34.27$ & $119.62 \pm 33.15$ & 0.142 \\
\hline High-density lipoprotein [mg/dl] & $34.68 \pm 8.63$ & $36.14 \pm 11.11$ & 0.186 \\
\hline Triglycerides [mg/dl] & $158.33 \pm 91.16$ & $140.88 \pm 95.88$ & 0.078 \\
\hline
\end{tabular}

*Mann-Whitney U-test; For other statistics Student's t-test.
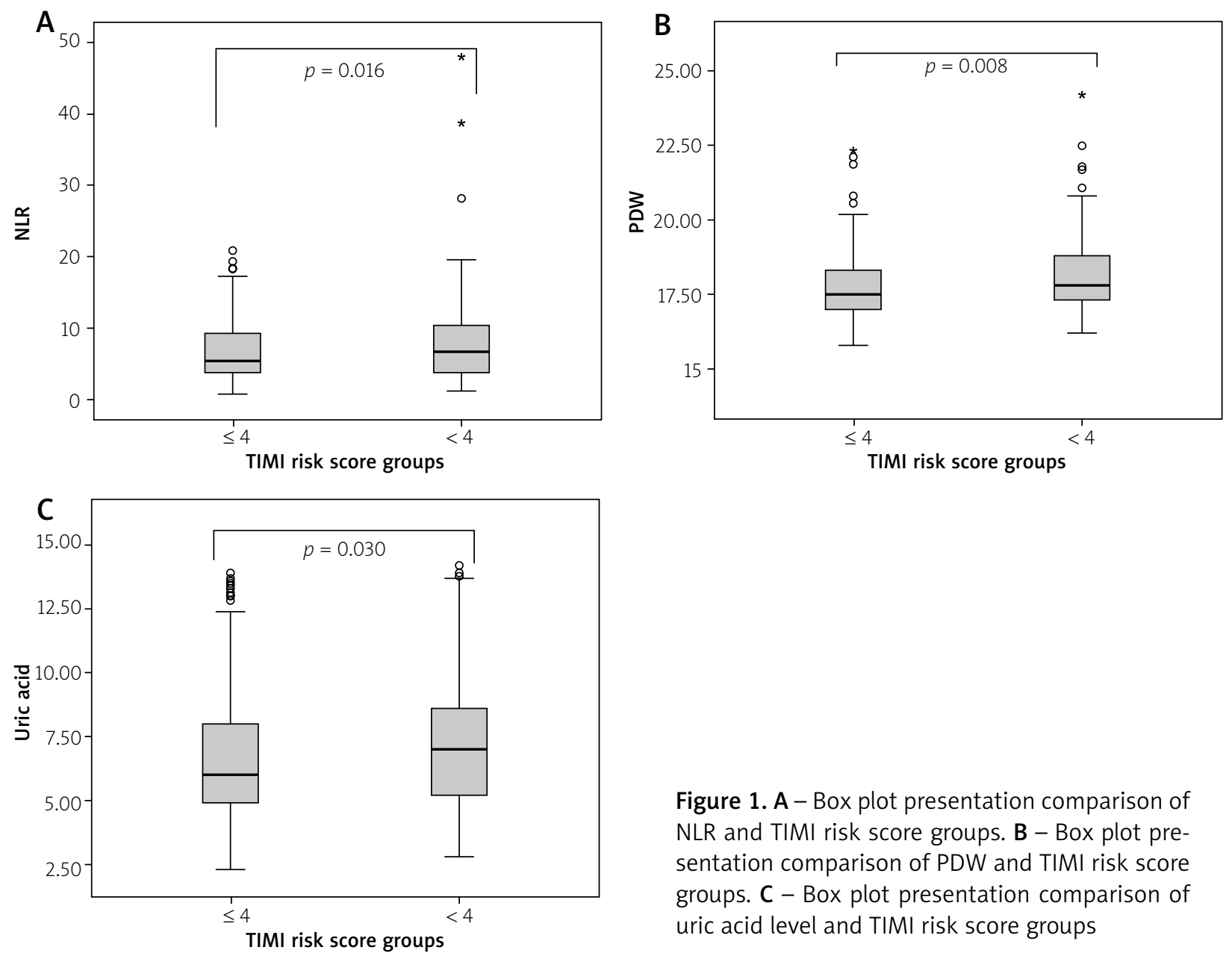

Figure 1. A-Box plot presentation comparison of NLR and TIMI risk score groups. B - Box plot presentation comparison of PDW and TIMI risk score groups. C - Box plot presentation comparison of uric acid level and TIMI risk score groups 
Table III. Major adverse cardiovascular events according to TIMI-STEMI risk score groups

\begin{tabular}{lccc}
\multicolumn{1}{c}{ Variables } & TIMI $\leq \mathbf{4}$ points & TIM > 4 points & Value of $\boldsymbol{p}$ \\
\hline In-hospital MACE, $n(\%)$ & $36(14)$ & $59(43)$ & $<0.001$ \\
\hline Advanced heart failure, $n(\%)$ & $6(2)$ & $20(15)$ & $<0.001$ \\
\hline Advanced pulmonary edema, $n(\%)$ & $4(2)$ & $15(11)$ & $<0.001$ \\
\hline Cardiogenic shock, $n(\%)$ & $7(3)$ & $26(19)$ & $<0.001$ \\
\hline Acute stent thrombosis, $n(\%)$ & $8(3)$ & $3(2)$ & $0.753^{*}$ \\
\hline Complete AVB requiring transient pacemaker, $n(\%)$ & $11(4)$ & $11(8)$ & 0.140 \\
\hline Serious ventricular arrhythmia, $n(\%)$ & $10(4)$ & $23(17)$ & $<0.001$ \\
\hline In-hospital mortality, $n(\%)$ & $11(4)$ & $33(24)$ & $<0.001$
\end{tabular}

*Fisher's exact test; For other statistics $\chi^{2}$ test; MACE - major adverse cardiovascular event, AVB - atrioventricular block.

\section{Discussion}

We showed that hematologic indices such as NLR, PDW and UA level were significantly associated with TIMI risk score for STEMI patients. Also, we demonstrated that admission LVEF, duration of chest pain, duration of hospitalization, Gensini and GRACE risk scores were significantly associated with TIMI risk score. Moreover, TIMI risk score and NLR, PDW and admission LVEF are significantly correlated. We found that there was a positive correlation of NLR and negative correlation of LVEF with TIMI risk score. Furthermore, LVEF was independently associated with TIMI risk score for STEMI. However, TIMI score was significantly associated with in-hospital MACE and in-hospital mortality in STEMI patients undergoing PPCI. Moreover, a TIMI score of greater than 4 was the cutoff point for predicting higher in-hospital mortality with a sensitivity of $75 \%$ and a specificity of $70 \%$.

Effective risk stratification is integral to the management of patients with ACS [25]. Even among patients with STEMI, for whom initial therapeutic options are well defined, patient risk characteristics have an effect on early therapeutic decision making $[6,26,27]$. In addition, increasing economic pressures have intensified the need for appropriate triage and clinical resource utilization, including decisions regarding transfer to tertiary centers [28]. In particular, the capacity to reliably identify patients at very low risk for fatal recurrent events may offer the opportunity to select low-risk patients for early hospital discharge $[29,30]$. Consequently, tools that enhance the clinician's ability to rapidly and accurately assess risk are of substantial interest. There are few models that have integrated weighing information from multivariate regression in a fashion similar to the TIMI risk score. Currently, there are no models which predict the short-term outcomes in STEMI [31].

A potentially relevant issue in the treatment of patients with STEMI is that this population is highly heterogeneous regarding their risk of adverse events. Thus, their correct stratification becomes essential in evaluating their prognosis and making accurate therapeutic decisions. An ideal risk score must be useful, simple and fast in order to predict short- and long-term prognosis

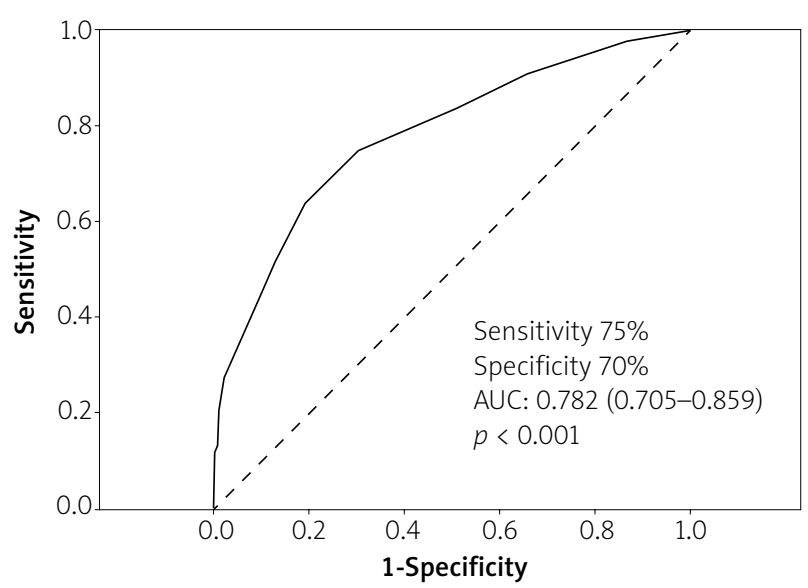

Figure 2. Receiver operator characteristic curve analysis of high TIMI risk score in predicting inhospital mortality

$[32,33]$. The TIMI risk score for STEMI is a clinical stratification calculated with data obtained at hospital presentation that can easily classify low and high risk patients. This risk score revealed that about $20 \%$ of patients were at higher risk of death [34]. The analysis was subsequently validated in an unselected patient population in the National Registry of Myocardial Infarction [7] and showed a strong predictive value for mortality in patients treated with thrombolytic therapy [35].

The TIMI risk score for STEMI serves as a prognostic calculator that discriminates high-risk patients with the combination of baseline variables that are part of the routine medical evaluation [11]. The TIMI risk score for STEMI has a strong association with mortality at 30 days. Patients with a score $>8$ points had a 40 -fold increase in mortality. At the high end, a score $>5$ points identified $12 \%$ of patients with a mortality risk $>2$-fold higher than the mean for the population. In contrast, the $12 \%$ of patients with a risk score of 0 had a mortality rate $<1 \%$ [11]. The TIMI risk score for STEMI is used for objective risk stratification of patients into one of two groups: low ( $\leq 4$ points); and high ( $>4$ points). The increased score is 
associated with higher in-hospital mortality and adverse cardiovascular events [36]. Thus, in our study we categorized the patients as having either a low ( $\leq 4$ points) or a high ( $>4$ points) score.

In our study of 390 patients who underwent $\mathrm{PPCl}$, $35 \%(n=138)$ were stratified as having a high risk score before the procedure. Thune et al. in the DANish trial in Acute Myocardial Infarction-2 (DANAMI-2) stratified patients by TIMI risk score; $25 \%$ of patients were high risk (TIMI score > 4 points) [37]. Lev et al., without identifying a high-risk group, reported that stratification with the TIMI risk score in patients undergoing $\mathrm{PPCl}$ predicts mortality and MACE (death, myocardial infarction, target vessel revascularization) [38].

We applied the TIMI risk score for STEMI in a group of patients who underwent $\mathrm{PPCl}$ and found that an increase in TIMI risk score is associated with increased frequency of in-hospital death. The TIMI risk score was developed to predict mortality. An important clinical implication of this study is that it identified a group of high-risk patients, who not only have a mortality rate higher than the low-risk group, but also have an increased frequency of in-hospital adverse events such as heart failure, development of cardiogenic shock, ventricular arrhythmias and advanced pulmonary edema.

The TIMI risk score for STEMI has been validated prospectively in various studies. The TIMI risk score for STEMI reliably identifies patients at very high risk while maintaining good discriminatory capacity in the low-risk range, where smaller absolute differences are more likely to impact clinical decisions. The TIMI risk scores include variables such as age, DM/HT or angina, heart rate $<100 \mathrm{bpm}$, systolic blood pressure $<100 \mathrm{~mm} \mathrm{Hg}$, Killip class II-IV, weight $<67 \mathrm{~kg}$, anterior MI or LBBB presentation, and latency $>4 \mathrm{~h}$, but do not include inflammatory markers.

\section{NLR and TIMI risk score for STEMI}

Several studies have examined the role of neutrophils and lymphocytes in modulating the inflammatory response to myocardial injury $[39,40]$. The NLR is a novel prognostic inflammatory marker $[13,14]$. Recently several studies have demonstrated that NLR is a marker of CVD prognosis [14, 41, 42]. Arbel et al. found that increased NLR was associated with increased severity of $C A D$, thereby providing additive predictive value to conventional risk factors and commonly used biomarkers e.g. C-reactive protein (CRP) and total WBC count [43].

Polat et al. reported that increased NLR was significantly associated with increased severity of rheumatic mitral valvular disease [44]. Yildiz et al. reported that increased NLR was independently and significantly associated with ventricular premature contraction existence [45].

We tested the predictive value of NLR with a well-validated standardized $\mathrm{MI}$ risk score. We have shown that NLR is significantly associated with TIMI-STEMI risk score. Multiple ACS studies now support the use of NLR as an admission biomarker, which can be used to determine prognosis $[46,47]$. The NLR can be readily calculated at point of care, thereby facilitating short- and long-term risk prediction for STEMI patients, even prior to revascularization. To our knowledge, the relationship between the NLR and TIMI risk score for STEMI has not been investigated. Our results demonstrate for the first time the predictive value of the TIMI risk score, STEMI and NLR. We found that high NLR was significantly positively correlated with TIMI risk score for STEMI.

\section{PLR and TIMI risk score for STEMI}

Platelets play an important role in the pathogenesis of ACS [16], which is caused by complex interactions between leukocytes and platelets. This results in the production of reactive oxygen species contributing to ischemic endothelial damage [48]. Prior studies have demonstrated that a low lymphocyte count in patients with acute MI and chronic CAD gives information about worse prognosis [49]. Platelet to lymphocyte ratio is derived from the number of platelets and lymphocytes. The prognostic significance of the PLR has been demonstrated in patients with some cancers $[19,20]$. It has been proposed to be a pro-thrombotic and inflammatory marker [50, 51]. Azab et al. reported that higher PLR values were associated with increased long-term mortality in non-STEMI patients [50]. Yildiz et al. found that high preprocedural PLR and NLR levels were significant and independent predictors of no reflow in patients undergoing PPCI [52]. Sunbul et al. determined that the PLR was a significant predictor of being a "non-dipper" or a hypertensive patient who sustains high blood pressures throughout the night time [53]. However, to the best of our knowledge, the relationship between the PLR and TIMI risk score for STEMI has not been investigated before. We found that high PLR was not significantly correlated with TIMI risk score for STEMI.

\section{PDW and TIMI risk score for STEMI}

Platelet distribution width (PDW) is a direct measurement of the variability in platelet size and is a marker of platelet activation [54]. In a recently conducted study, along with the demonstration of a relationship between CAD and PDW, there was also a significant relationship found between PDW and saphenous vein graft patency in patients who had coronary artery bypass operations [55]. Vagdatli et al. [56] reported that PDW is a more specific indicator of platelet activation than mean platelet volume (MPV), since there was no platelet swelling. The PDW directly measures the variability in platelet size and is a marker of platelet activation [57]. In a study with diabetic patients, Jindal et al. found a significant relationship between increased PDW and microvascular dysfunction [58]. Khandekar et al. found that PDW was significant- 
ly higher in patients with acute $\mathrm{MI}$ and unstable angina pectoris [59]. We found that high PDW was significantly correlated with an increased TIMI risk score for STEMI. To our knowledge, the relationship between the PDW and TIMI risk score for STEMI has not been investigated before. Our results demonstrate the relationship between TIMI risk score for STEMI and PDW.

\section{Uric acid and TIMI risk score for STEMI}

Uric acid levels are closely associated with inflammatory markers such as CRP [60]. Previous studies have shown that there was a significant relationship between UA levels and CAD [61-63], endothelial dysfunction [64], coronary reserve [65], coronary blood flow in patients undergoing elective angiography [66] and in STEMI patients undergoing PPCI [67]. Bickel et al. [61] demonstrated that UA is an independent predictor of mortality in patients with CAD. Bos et al. [62] reported that elevated UA levels are associated with an increased risk for acute MI. Recently UA levels were correlated with increased in-hospital MACE [67]. Lazzeri et al. [63] reported that UA is a prognostic indicator of in-hospital mortality in acute STEMI patients. In our study, we found that elevated UA levels were significantly associated with TIMI risk score for STEMI. To our knowledge, only one study by Wildi et al. has investigated the relationship between UA level and TIMI risk score for suspected MI [68]. Specifically, they investigated the relationship between UA levels and TIMI risk score in 143 patients (26\% STEMI, 75\% nonSTEMI). They found that UA values at presentation were associated with cardiovascular risk factors.

In our study, we found that NLR and UA, which are markers of inflammation, and PDW, which is a marker of platelet activation, are significantly associated with TIMI risk score in the STEMI patients before PPCI, but PLR is not significantly associated with TIMI risk score. We suggest that NLR, PDW and UA biomarkers, which are easily and cheaply measured, may be used in risk stratifying STEMI patients before PPCI. To our knowledge, this is the first study to report the joint relationship between UA level, PLR, NLR and PDW with TIMI risk score for STEMI before $\mathrm{PPCl}$. Our data included biochemical blood samples analyzed at admission, before starting any medication. We analyzed these blood samples prior to starting medication because WBC, its subtypes and platelets could be affected by infectious disorders, anxiety, and medication. Time of admission is the most critical time for clinicians to determine the choice of reperfusion therapy based on the predicted interventional outcome of the presenting case. Pre-admission or initial presentation prognostic indices are of vital importance in the accurate triage of emergency room patients for STEMI revascularization.

The limitations of the present study were a retrospective design and single-center experience. We could not compare NLR, PLR with other inflammatory markers, such as CRP, fibrinogen, or myeloperoxidase, because they were not routinely obtained in our study population.

\section{Conclusions}

Neutrophil to lymphocyte ratio, PDW and UA level are convenient, inexpensive and reproducible biomarkers for STEMI prognosis before PPCI. They can be utilized as robust stand-alone prognostic indicators for patients presenting with STEMI, even before accounting for eventual angiographic findings and outcomes. When these indicators are combined with standardized clinical mortality risk prediction scores, they markedly augment the predictive power of TIMI risk score. The interaction of the TIMI-STEMI risk score with these prognostic measures may be an area of interest for future investigation. We believe that these significant findings of our analysis can guide further clinical practice.

\section{Conflict of interest}

The authors declare no conflicts of interest.

\section{References}

1. Lopez AD, Mathers CD, Ezzati M, et al. Global and regional burden of disease and risk factors, 2001: systematic analysis of population health data. Lancet 2006; 367: 1747-57.

2. Ertaș F, Can Ö, Acet H, et al. The clinical significance of anticardiolipin antibody levels in patients with acute myocardial infarction: a regional study. Postep Kardiol Inter 2013; 9: 328-31.

3. Libby P. Inflammation in atherosclerosis. Nature 2002; 420: 868-74.

4. Pearson TA, Mensah GA, Alexander RW, et al. Markers of inflammation and cardiovascular disease: application to clinical and public health practice. Circulation 2003; 107: 499-511.

5. Zairis MN, Lyras AG, Bibis GP, et al. Association of inflammatory biomarkers and cardiac troponin I with multifocal activation of coronary artery tree in the setting of non-ST-elevation acute myocardial infarction. Atherosclerosis 2005; 182: 161-7.

6. Fibrinolytic Therapy Trialists' (FTT) Collaborative Group. Indications for fibrinolytic therapy in suspected acute myocardial infarction: collaborative overview of early mortality and major morbidity results from all randomized trials of more than 1000 patients. Lancet 1994; 343: 311-22.

7. Keeley EC, Boura JA, Grines CL. Primary angioplasty versus intravenous thrombolytic therapy for acute myocardial infarction: a quantitative review of 23 randomised trials. Lancet 2003; 361 : 13-20.

8. Thune JJ, Hoefsten DE, Lindholm MG, et al. Simple risk stratification at admission to identify patients with reduced mortality from primary angioplasty. Circulation 2005; 112: 2017-21.

9. Addala S, Grines CL, Dixon SR, et al. Predicting mortality in patients with ST-elevation myocardial infarction treated with primary percutaneous coronary intervention (PAMI risk score). Am J Cardiol 2004; 93: 629-32.

10. Halkin A, Singh M, Nikolsky E, et al. Prediction of mortality after primary percutaneous coronary intervention for acute myocardial infarction: the CADILLAC risk score. J Am Coll Cardiol 2005; 45: 1397-405. 
11. Morrow DA, Antman EM, Charlesworth A, et al. TIMI risk score for ST-elevation myocardial infarction: a convenient, bedside, clinical score for risk assessment at presentation: an intravenous nPA for treatment of infarcting myocardium early II trial substudy. Circulation 2000; 102: 2031-7.

12. The InTIME-II Investigators (2000). Intravenous npa for the treatment of infarcting myocardium early; intime-II, a double-blind comparison of single-bolus lanoteplase vs accelerated alteplase for the treatment of patients with acute myocardial infarction. Eur Heart J 2000; 21: 2005-13.

13. Kaya H, Ertas F, Islamoglu Y, et al. MS Association between neutrophil to lymphocyte ratio and severity of coronary artery disease. Clin Appl Thromb Hemost 2014; 20: 50-4.

14. Acet $\mathrm{H}$, Ertaş F, Akıl MA, et al. New inflammatory predictors for non-valvular atrial fibrillation: echocardiographic epicardial fat thickness and neutrophil to lymphocyte ratio. Int J Cardiovasc Imaging 2014; 30: 81-9.

15. Wagner DD, Burger PC. Platelets in inflammation and thrombosis. Arterioscler Thromb Vasc Biol 2003; 23: 2131-7.

16. Elsenberg $\mathrm{EH}$, van Werkum JW, van de Wal RM, et al. The influence of clinical characteristics, laboratory and inflammatory markers on 'high on-treatment platelet reactivity' as measured with different platelet function tests. Thromb Haemost 2009; 102: 719-27.

17. Zouridakis EG, GarciaMoll X, Kaski JC. Usefulness of the blood lymphocyte count in predicting recurrent instability and death in patients with unstable angina pectoris. Am J Cardiol 2000; 86: 449451

18. Ommen SR, Hammill SC, Gibbons RJ. The relative lymphocyte count predicts death in patients receiving implantable cardioverter defibrillators. Pacing Clin Electrophysiol 2002; 25 : 1424-8.

19. Smith RA, Ghaneh P, Sutton R, et al. Prognosis of resected ampullary adenocarcinoma by preoperative serum CA19-9 levels and platelet-lymphocyte ratio. J Gastrointest Surg 2008; 12 : 1422-8.

20. Wang D, Yang JX, Cao DY, et al. Preoperative neutrophil-lymphocyte and platelet-lymphocyte ratios as independent predictors of cervical stromal involvement in surgically treated endometrioid adenocarcinoma. Onco Targets Ther 2013; 6: 211-6.

21. Myocardial infarction redefined: a consensus document of The Joint European Society of Cardiology/American College of Cardiology Committee for the redefinition of myocardial infarction. Eur Heart J 2000; 21: 1502-13.

22. Killip T, Kimball JT. Treatment of myocardial infarction in a coronary care unit. A two year experience with 250 patients. Am J Cardiol 1967; 20: 457-64.

23. Granger CB, Goldberg RJ, Dabbous O, et al. Predictors of hospital mortality in the global registry of acute coronary events. Arch Intern Med 2003; 163: 2345-53.

24. Gensini GG. A more meaningful scoring system for determining the severity of coronary heart disease. Am J Cardiol 1983; 51: 606.

25. Maseri A, Rebuzzi AG, Cianflone D. Need for a composite risk stratification of patients with unstable coronary syndromes tailored to clinical practice. Circulation 1997; 96: 4141-2.

26. Becker RC, Burns M, Gore JM, et al. Early assessment and in-hospital management of patients with acute myocardial infarction at increased risk for adverse outcomes: a nationwide perspective of current clinical practice: the National Registry of
Myocardial Infarction (NRMI-2) Participants. Am Heart J 1998; 135: 786-96.

27. Hochman JS, Sleeper LA, Webb JG, et al. Early revascularization in acute myocardial infarction complicated by cardiogenic shock: SHOCK Investigators: should we emergently revascularize occluded coronaries for cardiogenic shock. N Engl J Med 1999; 341: 625-34.

28. Mark DB. Medical economics in cardiovascular medicine. In: Textbook of cardiovascular medicine. Topol EJ (ed.) Pa: Lippincott-Raven, Philadelphia 1998; 1033-61.

29. Newby LK, Califf RM, Guerci A, et al. Early discharge in the thrombolytic era: an analysis of criteria for uncomplicated infarction from the Global Utilization of Streptokinase and t-PA for Occluded Coronary Arteries (GUSTO) trial. J Am Coll Cardiol 1996; 27: 625-32.

30. Antman EM, Kuntz KM. The length of the hospital stay after myocardial infarction. N Engl J Med 2000; 342: 808-10.

31. Jacobs DR Jr, Kroenke C, Crow R, et al. PREDICT: a simple risk score for clinical severity and long-term prognosis after hospitalization for acute myocardial infarction or unstable angina: the Minnesota heart survey. Circulation 1999; 100: 599-607.

32. Rizo G, Ramirez J, Perez D, et al. Valor predictivo de muerte y complicaciones intrahospitalarias de los modelos de estratificación de riesgo en pacientes con infarto miocárdico agudo. Rev Fed Arg Cardiol 2011; 40: 57-64.

33. Zapata G. Predicción temprana del riesgo en el Infarto agudo de miocardio: una difícil tarea de todos los días. Rev Fed Arg Cardiol 2011; 40: 1-2.

34. Morrow DA, Antman EM, Charlesworth A, et al. TIMI risk score for ST-elevation myocardial infarction, a convenient, bedside, clinical score for risk assessment at presentation: an intravenous Npa for treatment of infarcting myocardium early II trial substudy. Circulation 2000; 102: 2031-7.

35. Morrow DA, Antman EM, Person L, et al. Application of TIMI risk score for ST elevation myocardial infarction in the National Registry of Myocardial Infarction 3. JAMA 2001; 286: 1356-9.

36. González-Pacheco H, Arias-Mendoza A, Álvarez-Sangabriel A, et al. The TIMI risk score for STEMI predicts in-hospital mortality and adverse events in patients without cardiogenic shock undergoing primary angioplasty. Arch Cardiol Mex 2012; 82: 7-13.

37. Thune JJ, Hoefsten DE, Lindholm MG, et al. Simple risk stratification at admission to identify patients with reduced mortality from primary angioplasty. Circulation 2005; 112: 2017-21.

38. Lev El, Kornowski R, Vaknin-Assa $H$, et al. Comparison of the predictive value of four different risk scores for outcomes of patients with ST-elevation acute myocardial infarction undergoing primary percutaneous coronary intervention. Am J Cardiol 2008; 102: 6-11.

39. Duffy BK, Gurm HS, Rajagopal V, et al. Usefulness of an elevated neutrophil to lymphocyte ratio in predicting long-term mortality after percutaneous coronary intervention. Am J Cardiol 2006; 97: 993-6.

40. Anderson JL, Ronnow BS, Horne BD, et al. Usefulness of a complete blood count derived risk score to predict incident mortality in patients with suspected cardiovascular disease. Am J Cardiol 2007; 99: 169-74.

41. Sen N, Afsar B, Ozcan F, et al. The neutrophil to lymphocyte ratio was associated with impaired myocardial perfusion and long term adverse outcome in patients with ST-elevated myocardial infarction undergoing primary coronary intervention. Atherosclerosis 2013; 228: 203-10. 
42. Isik T, Ayhan E, Uyarel H, et al. Association of neutrophil to lymphocyte ratio with presence of isolated coronary artery ectasia. Turk Kardiyol Dern Ars 2013; 41: 123-30.

43. Arbel YFA, Halkin A, Birati EY, et al. Neutrophil/lymphocyte ratio is related to the severity of coronary artery disease and clinical outcome in patients undergoing angiography. Atherosclerosis 2012; 225: 456-60.

44. Polat N, Yildiz A, Yuksel M, et al. Association of neutrophil-lymphocyte ratio with the presence and severity of rheumatic mitral valve stenosis. Clin Appl Thromb Hemost 2014; 20: 793-8.

45. Yildiz A, Oylumlu M, Yuksel $M$, et al. The association between the neutrophil-to-lymphocyte ratio and the presence of ventricular premature contractions in young adults. Clin Appl Thromb Hemost 2013 Nov 6 [Epub ahead of print] DOI: 10.1177/ 1076029613509478.

46. D’Ascenzo F, Biondi-Zoccai G, Moretti C, et al. TIMI, GRACE and alternative risk scores in Acute Coronary Syndromes: a metaanalysis of 40 derivation studies on 216,552 patients and of 42 validation studies on 31,625 patients. Contemp Clin Trials 2012; 33: 507-14.

47. Brkovic $V$, Dobric $M$, Beleslin B, et al. Additive prognostic value of the SYNTAX score over GRACE, TIMI, ZWOLLE, CADILLAC and PAMI risk scores in patients with acute ST-segment elevation myocardial infarction treated by primary percutaneous coronary intervention. Int J Cardiovasc Imaging 2013; 29: 1215-28.

48. Rezkalla SH, Kloner RA. No-reflow phenomenon. Circulation 2002; 105: 656-62.

49. Ommen SR, Gibbons RJ, Hodge DO, et al. Usefulness of the lymphocyte concentration as a prognostic marker in coronary artery disease. Am J Cardiol 1997; 79: 812-4.

50. Azab B, Shah N, Akerman M, et al. Value of platelet/lymphocyte ratio as a predictor of all cause mortality after non ST elevation myocardial infarction. J Thromb Thrombolysis 2012; 34: 326-34.

51. Bhatti I, Peacoc O, Lloyd G, et al. Preoperative hematologic markers as independent predictors of prognosis in resected pancreatic ductal adenocarcinoma: neutrophil lymphocyte versus platelet lymphocyte ratio. Am J Surg 2010; 200: 197203.

52. Yildiz A, Yuksel M, Oylumlu M, et al. The utility of the platelet-lymphocyte ratio for predicting no reflow in patients with ST-segment elevation myocardial infarction. Clin Appl Thromb Hemost 2015; 21: 223-8.

53. Sunbul M, Gerin F, Durmus E, et al. Neutrophil to lymphocyte and platelet to lymphocyte ratio in patients with dipper versus non-dipper hypertension. Clin Exp Hypertens 2014; 36: 217-21.

54. Herve P, Humbert M, Sitbon O, et al. Pathobiology of pulmonary hypertension: the role of platelets and thrombosis. Clin Chest Med 2001; 22: 451-8.

55. Ege MR, Guray U, Guray Y, et al. Platelet distribution width and saphenous vein disease in patients after CABG: association with graft occlusion. Herz 2013; 38: 197-201.

56. Vagdatli E, Gounari E, Lazaridou E, et al. Platelet distribution width: a simple, practical and specific marker of activation of coagulation. Hippokratia 2010; 14: 28-32.

57. Saya S, Hennebry TA, Lozano P, et al. Coronary slow flow phenomenon and risk for sudden cardiac death due to ventricular arrhythmias: a case report and review of literature. Clin Cardiol 2008; 31: 352-5.

58. Jindal S, Gupta S, Gupta R, et al. Platelet indices in diabetes mellitus: indicators of diabetic microvascular complications. Hematology 2011; 16: 86-9.
59. Khandekar MM, Khurana AS, Deshmukh SD, et al. Platelet volume indices in patients with coronary artery disease and acute myocardial infarction: an Indian scenario. J Clin Pathol 2006; 59: 146-9.

60. Frohlich M, Imhof A, Berg G, et al. Association between C-reactive protein and features of the metabolic syndrome: a population-based study. Diabetes Care 2000; 23: 1835-9.

61. Bickel C, Rupprecht HJ, Blankenberg S, et al. Serum uric acid as an independent predictor of mortality in patients with angiographically proven coronary artery disease. Am J Cardiol 2002; 89: 12-7.

62. Bos MJ, Koudstaal PJ, Hofman A, et al. Uric acid is a risk factor for myocardial infarction and stroke: the Rotterdam study. Stroke 2006; 37: 1503-7.

63. Lazzeri C, Valente S, Chiostri M, et al. Uric acid in the acute phase of ST elevation myocardial infarction submitted to primary PCI: its prognostic role and relation with inflammatory markers: a single center experience. Int J Cardiol 2010; 138: 206-9.

64. Erdogan D, Gullu H, Caliskan M, et al. Relationship of serum uric acid to measures of endothelial function and atherosclerosis in healthy adults. Int J Clin Pract 2005; 59: 1276-82.

65. Erdogan D, Gullu H, Caliskan M, et al. Coronary flow reserve and coronary microvascular functions are strongly related to serum uric acid concentrations in healthy adults. Coron Artery Dis 2006; 17: 7-14.

66. Yildiz A, Yilmaz R, Demirbag R, et al. Association of serum uric acid level and coronary blood flow. Coron Artery Dis 2007; 18: 607-1

67. Akpek M, Kaya MG, Uyarel H, et al. The association of serum uric acid levels on coronary flow in patients with STEMI undergoing primary $\mathrm{PCl}$. Atherosclerosis 2011; 219: 334-41.

68. Wildi K, Haaf P, Reichlin T, et al. Uric acid for diagnosis and risk stratification in suspected myocardial infarction. Eur J Clin Invest 2013; 43: 174-82 